This is a pre-print version.

Published in: Journal of Sound and Vibration (2018) Vol. 434, pp. 1-16.

DOI: $10.1016 / j . j s v .2018 .07 .022$.

\title{
Aeroacoustic response of an array of tubes with and without bias-flow
}

Aswathy Surendran ${ }^{\mathrm{a}}$, Maria A. Heckl ${ }^{\mathrm{a}, *}$, Luck Peerlings ${ }^{\mathrm{b}}$, Susann Boij ${ }^{\mathrm{b}}$, Hans Bodén ${ }^{\mathrm{b}}$, Avraham Hirschberg ${ }^{\mathrm{c}}$

${ }^{a}$ School of Chemical and Physical Sciences, Keele University, Staffordshire, United Kingdom, ST55BG

${ }^{b}$ Marcus Wallenberg Laboratory for Sound and Vibration Research, Aeronautical and Vehicle Engineering, KTH Royal Institute of Technology, SE-10044, Stockholm, Sweden

${ }^{c}$ Department of Applied Physics, Eindhoven University of Technology, 5600 MB Eindhoven, The Netherlands

\section{Abstract}

Heat exchangers, consisting of tube arrays in a cross-flow are a vital component of power generation systems. They are of interest from an acoustic point of view, because they can reflect, transmit and absorb an incident sound wave; in other words, they have the potential to act as a sound absorber and even as a passive control device to prevent a thermoacoustic instability in the power generation system. This paper presents a fundamental study of the aeroacoustic response of a tube array with and without bias-flow (also called crossflow). The study has a theoretical and an experimental side. On the theoretical side, a new model, based on the assumption of quasi-steady flow, was developed to predict the acoustic reflection and transmission coefficient of a tube array with bias-flow. Also, the model by Huang and Heckl (Huang and Heckl, 1993, Acustica 78, 191-200) for the case without bias-flow was evaluated. On the experimental side, flow-duct experiments using a multi-microphone technique were performed to validate the predictions from both models. The agreement was found to be very good for low frequencies. The measurements revealed the limit of validity of the quasi-steady model in terms of the Strouhal number. Although this limit is quite low, our quasi-steady model can serve as a valuable tool for designers of heat exchangers.

Keywords: aeroacoustics, low-frequency response, tube array, heat exchanger, low Mach number bias-flow, duct flow

\section{Introduction}

This paper investigates the acoustic response of an array of tubes (also referred to as a tube row). Tube rows are components in many engineering systems, where they may act as

\footnotetext{
*Corresponding author

Email address: m.a.heckl@keele.ac.uk (Maria A. Heckl)
} 
heat exchangers. A well-known example is the heat exchanger tubes in a domestic boiler (see Fig. 11. The main elements, from an acoustic point of view, are the combustion chamber, the flame, the tube row, and the cross-flow (or bias-flow) through the gaps between the tubes. The combustion chamber acts as an acoustic resonator, and the flame acts as a potential sound source, generating a high-amplitude acoustic field; the tube row scatters the acoustic waves, and the cross-flow introduces aerodynamic effects.

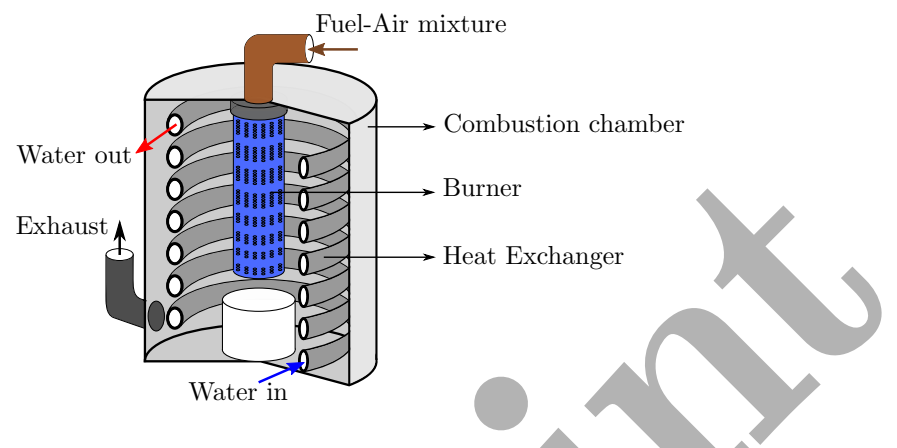

Figure 1: Schematic of domestic boiler system.

Altogether, this is a scenario where several physical effects occur simultaneously:

- thermoacoustic instabilities excited by the flame in the combustion chamber;

- reflection and transmission of acoustic waves impinging on the tube row;

- flow separation due to the cross-flow, leading to jet formation and vortex shedding downstream of the tube row, as well as to viscous and thermal boundary layers;

- feedback between the acoustic field and the aerodynamic effects, leading to synchronised vortex shedding;

- vibrations of the individual tubes, leading to structural resonances and losses.

Our work is motivated by the possibility that the tube row, together with the cross-flow, could provide a form of passive instability control. However, in order to investigate this possibility, it is necessary to have a model for the acoustic behaviour of the combined system, tube row plus cross-flow. The aim of our paper is to present such a model. We consider the simplest configuration: a tube row with or without bias-flow and an incident acoustic wave. We do not include thermoacoustic effects in our model, nor structural vibrations; in other words: we treat the fluid surrounding the tube row as homogeneous, and the "tubes" as solid rods. Our study is two-dimensional in the sense that the cross-flow and acoustic waves are perpendicular to the tubes. The tubes are all parallel and equally spaced. It should also be noted that in the present work, we consider only a single row of tubes (hence termed tube row) and not tube bank (or bundle). 
Wave scattering by a periodic array of scatterers is a phenomenon that occurs widely in physics and engineering. Examples are: water waves scattered by off-shore structures, electromagnetic waves diffracted by a wire grating, and acoustic waves used for non-destructive testing (NDT) purposes to examine periodic arrays of tubes immersed in a fluid. Studies involving acoustic waves have been performed by Mungur and Fahy [1] and Kristiansen and Fahy [2] who reduced the problem by treating the tube row as layer of a homogeneous medium with an effective density and speed of sound. Linton and Evans [3] used a multipole expansion method to describe the acoustic scattering of water waves by an array of rigid cylinders. Heckl et al. [4, 5] built on Twersky's grating theory [6, 7, 8, and developed expressions for the reflection and transmission coefficients of plane pressure waves impinging from arbitrary directions on a grating formed by fluid-filled flexible cylindrical tubes. In Huang and Heckl 9], they extended the model to include several loss mechanisms (structural losses in the flexible tube walls, viscous and thermal losses at the tube surfaces)

While the papers quoted in the previous paragraph are about tube rows without crossflow, there are also relevant studies for the case with cross-flow. Quinn and Howe [10] studied a row of infinitesimally thin rigid strips in cross-flow and calculated the attenuation of an incident sound wave; they found that the attenuation increases with decreasing Strouhal number; in other words, for a given tube diameter and frequency, the sound attenuation increases with increasing cross-flow velocity. However, in their approach they considered a highly idealised configuration (rigid strips instead of rigid tubes), so their results can be seen only as a qualitative indication. Dowling and Hughes [1] derived expressions for the reflection and transmission coefficient of a slit plate (equivalent to a row of rigid rectangular rods) in cross-flow. Their expressions show that the absorption coefficient increases with cross-flow velocity, and is largely independent of the frequency. However, all these studies mentioned in this paragraph rely on the Kutta condition, i.e. they assume that the scatterer has a sharp edge.

A configuration closely related to an array of cylinders in cross-flow is a single cylinder in a hard-walled flow-duct, because this can be modelled by the method of image sources. This configuration has been considered by the group of Hirschberg [12, 13, 14] in their work on modelling human voice production. They represented the wind-pipe by a rectangular duct, the vocal chords by two diaphragms (half-cylinders) stretched across the duct with a gap between them, and the air expelled from the lungs by a mean-flow. They used the quasi-steady theory, first proposed by Ronneberger [15] in their model. In quasi-steady theory, the combined flow field (cross-flow superimposed by acoustic wave) is treated as a 
succession of steady flows. This assumption is obviously valid for steady flows, but it also works well if the superimposed acoustic field has a low frequency [16, 17, 18. Using this approximation, Hofmans [19, 20, calculated the reflection and transmission coefficient of a sharp edged diaphragm in a flow-duct.

Still missing in the literature is a model, which makes it possible to calculate the reflection and transmission coefficient of a smooth acoustic scatterer, such as a cylinder with circular cross-section, in cross-flow. This is the gap we are trying to fill with this paper.

The modelling aspects and the theoretical background of our study are described in Section 2. This section is divided into two parts: in Section 2.1. we provide a brief summary of the Huang and Heckl model [9] for the acoustic response of tube array in the absence of flow; in Section 2.2, we develop a quasi-steady model for the acoustic response of the tube array in the presence of flow. We study two tube row geometries, both theoretically and experimentally; they are described in Section 3 , The theoretical predictions are given in Section 4 The experimental setup and procedure are presented in Section 5 Section 6 presents our experimental results and the validation of our model; it is again in two parts: Section 6.1 is for the tube row without cross-flow, and Section 6.2 is for the tube row with cross-flow. The limit of validity of the quasi-steady model is presented in Section 6.3, and the conclusions are given in Section 7

\section{Theoretical Background}

\subsection{Tube row without cross-flow}

Huang and Heckl 9] considered a row of infinitely long, periodically spaced tubes as shown in Fig. 2. Each tube has diameter $d$ and the tubes are equally spaced (spacing $h_{p}$ ), acting like a diffraction grating when a plane acoustic wave falls on it. Hence, they used the grating theory, initially proposed by Twersky [6, 7] for optical grating, to obtain the expressions for the reflection and transmission coefficients of this tube row.

The expressions for the transmitted and reflected pressure fields were found to be

$$
\begin{gathered}
p_{t}(r, \varphi)=\Psi_{0} e^{i k_{0} r \cos \left(\varphi-\varphi_{0}\right)}+\frac{2 \Psi_{0}}{k_{0} h_{p}} \sum_{\nu=-\infty}^{\infty} \frac{1}{\cos \varphi_{\nu}} \sum_{n=-\infty}^{\infty} A_{n} e^{i n \varphi_{\nu}+i k_{0} r \cos \left(\varphi-\varphi_{0}\right)} \\
p_{r}(r, \varphi)=\frac{2 \Psi_{0}}{k_{0} h_{p}} \sum_{\nu=-\infty}^{\infty} \frac{1}{\cos \varphi_{\nu}} \sum_{n=-\infty}^{\infty} A_{n} e^{i n\left(\pi-\varphi_{\nu}\right)-i k_{0} r \cos \left(\varphi+\varphi_{0}\right)}
\end{gathered}
$$

where $\Psi_{0}$ is the amplitude of the incident wave, $k_{0}$ is the incident wave number, $\varphi_{0}$ is the angle of incidence, $(r, \varphi)$ are the polar co-ordinates, $\nu$ is an integer to denote the scattering angle $\varphi_{\nu}$ and $A_{n}$ is the multiple scattering coefficient of the tube row. 


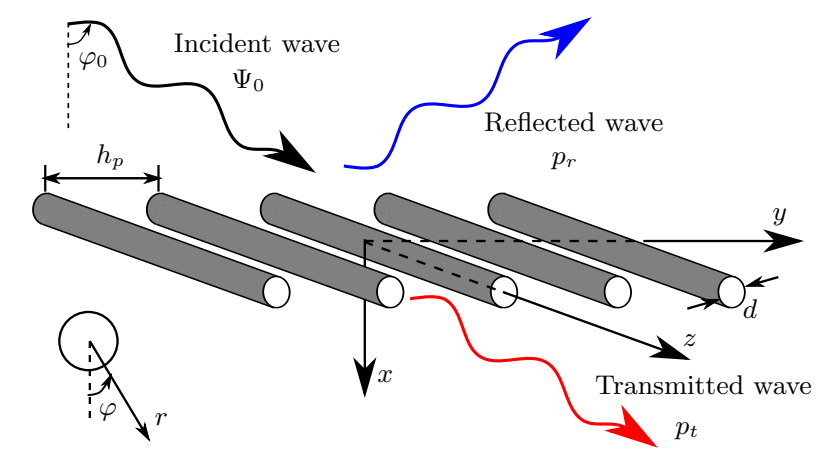

Figure 2: Geometry of the tube row.

The coefficient $A_{n}$ is evaluated from [7]:

$$
A_{n}=a_{n}\left[e^{-i n \varphi_{0}}+\sum_{m=-\infty}^{\infty} A_{m} F_{n-m}\right]
$$

where $a_{n}$ is the scattering coefficient of a single tube (given by Eq. (3) in Huang and Heckl [9]) and $F_{n-m}$ is the Schlömilch series. $F_{n-m}$ is a function of the incident angle $\varphi_{0}$ and the ratio of the spacing to the wavelength $\left(h_{p} / \lambda_{0}\right)$.

For normal incidence of the pressure wave $\left(\varphi_{0}=0\right)$, the transmission and reflection coefficients, $T$ and $R$ are written as the ratio of the transmitted and reflected waves to the incident wave, respectively. Hence,

$$
\begin{aligned}
T=1+\frac{2}{k_{0} h_{p}} \sum_{n=-\infty}^{\infty} A_{n}, \\
R=\frac{2}{k_{0} h_{p}} \sum_{n=-\infty}^{\infty} A_{n} e^{i n \pi} .
\end{aligned}
$$

\subsection{Tube row with cross-flow}

\subsubsection{Quasi-steady model}

Quasi-steady modelling was first proposed by Ronneberger [15]. This modelling approach is based on the assumption that the fluctuations in a time-dependent flow are slow enough to be treated as a succession of steady flows. We follow the approach by Hofmans [20] who applied quasi-steady modelling to rectangular scatterers in a flow duct. In our study, the scatterers are two half-cylinders, attached to opposite walls of a flow-duct, as shown in Fig. 3. The half-cylinders are separated by a gap of width $h_{g}$, and there is a bias-flow through the gap with velocity $u_{g}$. The subscript $g$ denotes gap. 
We define the following non-dimensional quantities in order to conduct an order of magnitude analysis and to describe the key assumptions made in our model.

$$
\begin{aligned}
\text { Strouhal number : } S t & =\frac{f}{u_{g} / r}=\frac{\text { flow time scale }}{\text { acoustic time scale }} \\
\text { Helmholtz number : } H e & =\frac{h_{d}}{c / f}=\frac{\text { duct height }}{\text { wavelength }} \\
\text { Mach number : } M & =\frac{u}{c}=\frac{\text { flow speed }}{\text { speed of sound }}
\end{aligned}
$$

where $f$ is frequency of the acoustic wave, $r$ is the radius of the cylinder, $h_{d}$ is the duct height, $c$ is the speed of sound, and $u$ is the velocity of the flow in the duct. We make the following assumptions:

(i) The Strouhal number, which is a measure of the relevance of the aerodynamic effects in the flow, is very small, i.e. $S t \ll 1$ (low frequency). This means that in a short time interval, acoustic waves can be regarded as "frozen", while aerodynamic effects evolve. In this regime, quasi-steady modelling is a valid approach.

(ii) The Helmholtz number is a measure of the compactness of the source/sink region. When we neglect wave propagation effects, we can assume that $(H e)^{2} \ll 1$ (see page 14 in 21]) This means that the acoustic wavelength is much longer than the cylinder cross-section; it is also much longer than the mixing zone downstream of the cylinder due to vortex shedding. It is therefore safe to assume that there are no phase changes to the acoustic quantities across the blocked region.

(iii) The Mach number is a measure of the importance of the convection effects in the flow domain. In order to maintain a subsonic flow through the gap $\left(M_{g}<1\right)$, we restrict our analysis to very low incoming Mach numbers. Also, we assume that the average properties like density and temperature are uniform across Region $j$ in Fig. 3 . These properties in a flow scale with $M^{2}$ and hence for them to be uniform, we assume that $M^{2} \ll 1$.

(iv) The flow within the duct is inviscid and compressible (unless stated otherwise).

Using these assumptions, we will now derive expressions for the aeroacoustic response of the two half-cylinders in the duct.

\subsubsection{Conservation Equations}

We consider the duct shown in Fig. 3 with cross-sectional height $h_{p}$ (considering the depth to be unity). The duct is divided into three regions. (a) Region 1 with uniform flow upstream of the cylinders. (b) Region $j$ (shaded region) containing the cylinders and a compact source/sink region around them, where acoustic energy could be produced or dissipated due to vortex shedding. The flow, after passing through the gap $\left(h_{g}\right)$ between 
the half-cylinders, separates from the cylinder surfaces forming a jet with cross-sectional height $h_{j}$. The jet is then followed by a short mixing region. (c) Region 2 with uniform flow downstream of the cylinders. The variables $p, u, \rho$ and $h$ in Fig. 3 denote pressure, velocity, density and cross-sectional height respectively, and the subscripts denote the corresponding region within the domain.

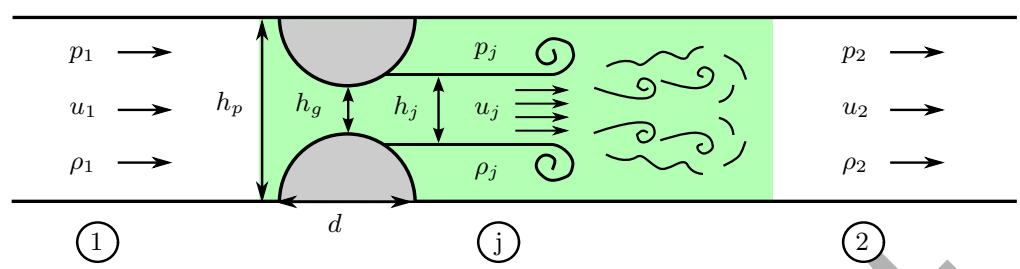

Figure 3: Schematic of the flow within the duct.

The flow from Region 1 into the jet is assumed to be isentropic and irrotational. Therefore, we can apply the continuity equation, the isentropic gas relation and the energy equation across regions 1 and $j$.

$$
\begin{aligned}
h_{p} \rho_{1} u_{1} & =h_{j} \rho_{j} u_{j} \\
\frac{p_{1}}{p_{j}} & =\left(\frac{\rho_{1}}{\rho_{j}}\right)^{\gamma} \\
\frac{1}{2} u_{1}^{2}+\left(\frac{\gamma}{\gamma-1}\right) \frac{p_{1}}{\rho_{1}} & =\frac{1}{2} u_{j}^{2}+\left(\frac{\gamma}{\gamma-1}\right) \frac{p_{j}}{\rho_{j}}
\end{aligned}
$$

where $\gamma$ is the ratio of specific heats.

Downstream of the jet, there is a turbulent mixing zone, where the jet's kinetic energy is dissipated while some pressure recovery occurs. We have assumed the jet mixing zone to be very small (see assumption (ii) in Section 2.2.1).

Beyond the mixing zone, the flow becomes uniform again (Region 2), but it is no longer isentropic. We use the continuity and momentum equations to describe the link between Region $j$ and Region 2.

$$
\begin{aligned}
h_{j} \rho_{j} u_{j} & =h_{p} \rho_{2} u_{2} \\
h_{p} p_{j}+h_{j} \rho_{j} u_{j}^{2} & =h_{p} p_{2}+h_{p} \rho_{2} u_{2}^{2}
\end{aligned}
$$

In our analysis, we have neglected the heat transfer, viscous and frictional losses at the walls. So, the conservation of energy across Region $j$ can be written as

$$
\frac{1}{2} u_{1}^{2}+\left(\frac{\gamma}{\gamma-1}\right) \frac{p_{1}}{\rho_{1}}=\frac{1}{2} u_{2}^{2}+\left(\frac{\gamma}{\gamma-1}\right) \frac{p_{2}}{\rho_{2}}
$$

However, we do take into account one important viscous flow effect: the flow separation on the cylinder surface. We focus on high Reynolds number flows $(R e>8000)$, where the 
viscous effects are confined to very thin boundary layers and shear layers, and the flow can be regarded as inviscid elsewhere. In order to determine the jet's cross-sectional height $h_{j}$, the flow separation point is required. We find this point by solving the von Kármán equations using the Thwaites' method. Details of the procedure for can be found in Appendix A. The procedure is valid when the boundary layer thickness $\delta_{b l}$ is much smaller than the gap between the cylinders, i.e. $\delta_{b l} \ll h_{g}$, where $\delta_{b l}=\sqrt{\nu r / u_{g}}$, and $\nu$ is the kinematic viscosity. In our study, this condition is satisfied: $\delta_{b l}=0.06 \mathrm{~mm}$ for $u_{1}=5 \mathrm{~m} / \mathrm{s}$ (the smallest velocity we are considering), and $h_{g} \approx 4 \cdots 5 \mathrm{~mm}$.

\subsubsection{Scattering Matrix}

In order to account for the wave convection effects, we use total enthalpy as the acoustic variable [12, 19, 22] (instead of acoustic pressure). Total enthalpy is a natural choice for an acoustic variable because it satisfies the inhomogeneous wave equation of convected type as shown by Doak [23]. It is defined as $H_{i}^{ \pm}=p_{i}^{ \pm}\left(1 \pm M_{i}\right) / \rho_{1}$, where the superscript "+" denotes waves travelling in the direction of the mean flow, and "-" denotes waves travelling in the opposite direction.

The scattering matrix relates the enthalpy perturbations travelling away from the scatterer to those travelling towards the scatterer. Our procedure to obtain the scattering matrix is similar to that given in Hofmans [20]. We split Eqs. (9) - (14) into two sets of equations: a nonlinear set for the steady flow and a linearised set for the acoustic perturbations, which consist of forward and backward travelling components, $p^{+}$and $p^{-}$, as shown in Fig. 4 . The steady-flow equations, which are similar to Eqs. (9) - (14), are solved first, and subsequently used in the equations for the acoustic perturbations.

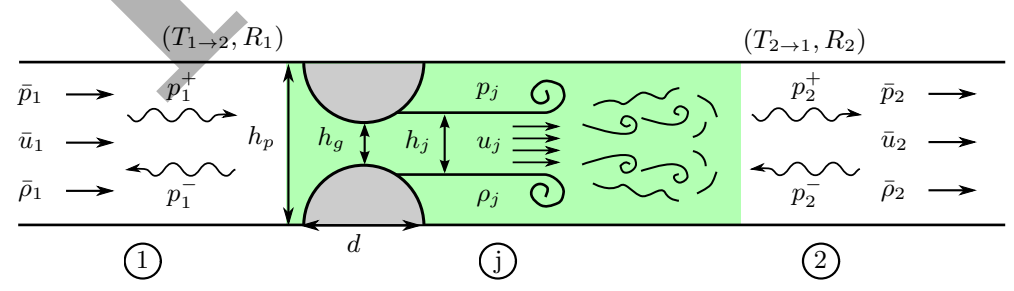

Figure 4: Schematic of the flow within the duct showing the mean quantities and the forward and backward travelling acoustic pressure waves in Regions 1 and 2.

The perturbation equations can be arranged in such a way that the outgoing enthalpy waves $\left(\left(1-M_{1}\right) p_{1}^{-}\right.$and $\left.\left(1+M_{2}\right) p_{2}^{+}\right)$are expressed in terms of the incoming enthalpy waves $\left(\left(1+M_{1}\right) p_{1}^{+}\right.$and $\left.\left(1-M_{2}\right) p_{2}^{-}\right)$; the matrix that relates them is the scattering matrix. Details 
of the derivation of this matrix equation, which reads

$$
\left[\begin{array}{c}
\left(1+M_{2}\right) p_{2}^{+} \\
\left(1-M_{1}\right) p_{1}^{-}
\end{array}\right]=\left[\begin{array}{cc}
T_{1 \rightarrow 2} & R_{2} \\
R_{1} & T_{2 \rightarrow 1}
\end{array}\right]\left[\begin{array}{l}
\left(1+M_{1}\right) p_{1}^{+} \\
\left(1-M_{2}\right) p_{2}^{-}
\end{array}\right]
$$

can be found in [24].

The matrix elements $T$ and $R$ denote, respectively, the transmission and reflection coefficient of the scatterer. For waves incident from the upstream side, the coefficients are denoted by $T_{1 \rightarrow 2}$ and $R_{1}$; for waves incident from the downstream side, the notation is $T_{2 \rightarrow 1}$ and $R_{2}$.

\section{Geometries studied}

In order to validate the models described in Section 2, we considered two samples:

1. an array of cylinders ( 5 full cylinders and 2 half-cylinders) of diameter $d=16 \mathrm{~mm}$, width $l=25 \mathrm{~mm}$, and spaced $h_{p}=20 \mathrm{~mm}$ apart (see Fig. 5 , left), and

2. two half-cylinders of diameter $d=20 \mathrm{~mm}$, width $l=120 \mathrm{~mm}$ and spaced $h_{p}=25 \mathrm{~mm}$ apart (see Fig. 5 , right).

The former sample is denoted as $d_{16}$ and the latter as $d_{20}$, where the subscript stands for the diameter in $m m$. The open area ratio of both samples was the same: $\eta=\left(h_{p}-d\right) / d=0.25$.
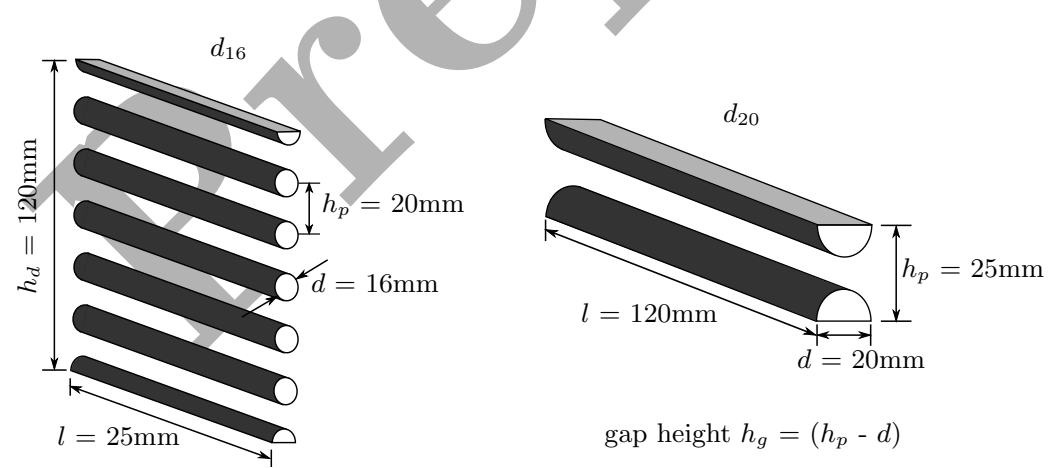

Figure 5: Schematic of the geometries studied (not to scale).

\section{Theoretical results for the quasi-steady model}

The reflection and transmission coefficients predicted from the quasi-steady model are shown in Fig. 6 for flow Mach numbers up to $M_{1}=0.12$ (higher values for $M_{1}$ would lead to transonic flow through the gap). The continuous curves give the results for the sample $d_{16}$, and the markers those for $d_{20}$. 


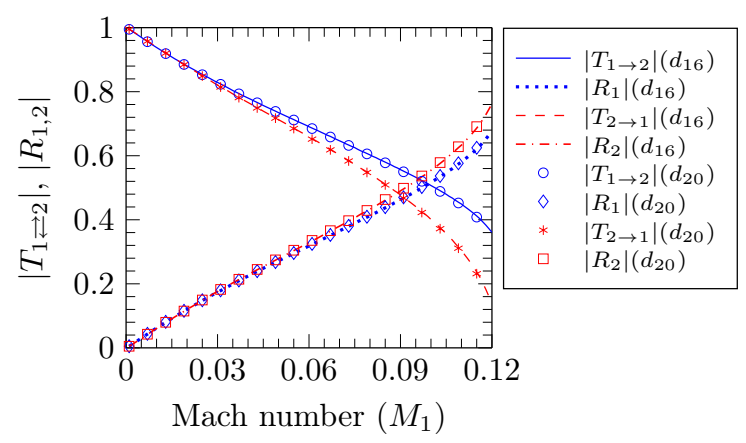

Figure 6: Quasi-steady model results for the two samples. The curves are for $d_{16}$ and the markers are for $d_{20}$.

We observe that the transmission coefficients decrease with Mach number, while the reflection coefficients increase. This is as expected because an increase in Mach number leads to a decrease in jet diameter, and this enhances the blockage to the flow. We also observe that the curves for $d_{16}$ are quite similar to those of $d_{20}$. Initially, for small Mach numbers, $\left|T_{1 \rightarrow 2}\right|$ and $\left|T_{2 \rightarrow 1}\right|$ (as well as $\left|R_{1}\right|$ and $\left|R_{2}\right|$ ) coincide, and the scattering matrix is symmetric. As the Mach number increases, the elements of the scattering matrix $\left|T_{1 \rightarrow 2}\right|$ and $\left|T_{2 \rightarrow 1}\right|$ (as well as $\left|R_{1}\right|$ and $\left|R_{2}\right|$ ) diverge more and more from each other, and the scattering matrix becomes asymmetric. This observation has also been made by Hofmans [20].

It is important to note that the curves for $\left|T_{1 \rightleftarrows 2}\right|$ and $\left|R_{1,2}\right|$ are the same irrespective of the samples chosen. This result indicates that the geometry can be scaled in terms of the open area ratio $\eta$.

\section{Experiments}

\subsection{Experimental setup}

The experimental setup is shown schematically in Fig. 7. It consisted of a long aluminium duct of rectangular cross-section $(120 \mathrm{~mm} \times 25 \mathrm{~mm})$ and with a wall thickness of $15 \mathrm{~mm}$. The samples were placed within the duct, one at a time. Acoustic excitation was provided by two pairs of loudspeakers placed near the upstream and downstream ends of the duct, far from the sample to ensure that only plane waves are travelling towards the sample. In order to reduce the acoustic reflections at the ends, the duct was connected to an anechoic chamber at the upstream end and to a muffler in the downstream end.

The pressure fluctuations up and downstream of the sample were recorded using eight flush mounted microphones (1/4" pre-polarised condenser microphones by G.R.A.S. Type 40BD), four on either side of the sample. The microphones were all calibrated in gain and phase, relative to each other. This was done using a calibrator, where all the microphones 
were subjected to the same sound field. The microphone signals passed through signal conditioners (Nexus conditioning amplifier, Type 2690-A-0S4) to the data acquisition system (Agilent E1421B VXI Main frame and Agilent E1432A Digitizer). The DAQ also acted as signal generator (Agilent E1434A Signal generator) for the loudspeakers; these were driven with a variable amplification, which was adjusted to the sound field in the duct. The flow velocity in the upstream end was measured using a static-pitot tube and a SWEMA3000 pressure transducer. Details of the setup and measurement procedure can be found in Zhou [25] and Peerlings [26].

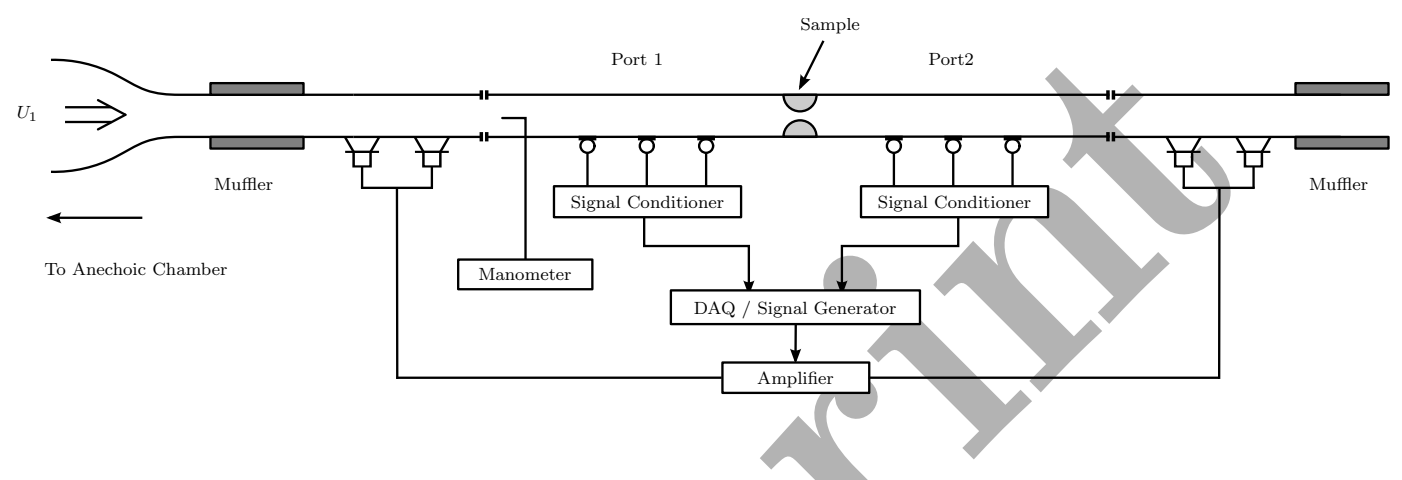

Figure 7: Schematic of the experimental setup.

\subsection{Experimental procedure}

The scattering matrix in Eq. 15 has been derived for the total enthalpy in the flow domain. However, in practical situations, it is more convenient to obtain the scattering matrix from acoustic pressure measurements. The scattering matrices for total enthalpy and pressure are related as shown in Eq. (16), where the scattering matrix on the left hand side, with the superscript ' $P$ ' for the transmission and reflection coefficients, denotes the scattering matrix for pressure. The transmission and reflection coefficients on the right hand side are those pertaining to the total enthalpy (elements of the scattering matrix in Eq. (15)).

$$
\left[\begin{array}{cc}
T_{1 \rightarrow 2}^{P} & R_{2}^{P} \\
R_{1}^{P} & T_{2 \rightarrow 1}^{P}
\end{array}\right]=\left[\begin{array}{cc}
T_{1 \rightarrow 2}\left(\frac{1+M_{1}}{1+M_{2}}\right) & R_{2}\left(\frac{1-M_{2}}{1+M_{2}}\right) \\
R_{1}\left(\frac{1+M_{1}}{1-M_{1}}\right) & T_{2 \rightarrow 1}\left(\frac{1-M_{2}}{1-M_{1}}\right)
\end{array}\right]
$$

The elements of the scattering matrix (for pressure) are determined from the measurements of two independent pressure fields [27, generated by upstream excitation (denoted by superscript A) and downstream excitation (denoted by superscript B). Altogether, this gives 4 linear equations for the 4 elements of the scattering matrix,

$$
\left[\begin{array}{cc}
p_{2}^{+A} & p_{2}^{+B} \\
p_{1}^{-A} & p_{1}^{-B}
\end{array}\right]=\left[\begin{array}{cc}
T_{1 \rightarrow 2}^{P} & R_{2}^{P} \\
R_{1}^{P} & T_{2 \rightarrow 1}^{P}
\end{array}\right]\left[\begin{array}{ll}
p_{1}^{+A} & p_{1}^{+B} \\
p_{2}^{-A} & p_{2}^{-B}
\end{array}\right]
$$


A two-port multi-microphone measurement technique [26, 28] was used to obtain the pressure data. The acoustic pressure field within the duct can be written as, assuming a time dependence of $e^{-i \omega t}$,

$$
p^{\prime}(x)=p^{+} \exp \left(i k^{+} x\right)+p^{-} \exp \left(-i k^{-} x\right)
$$

where $p^{\prime}(x)$ is the measured complex pressure at position $x$ and $k^{+}$and $k^{-}$are the forward and backward travelling wavenumbers. The wavenumbers are corrected for the visco-thermal losses as well as the convection effects as [29],

$$
k^{ \pm}=\frac{k_{0}+(1+i) \beta_{0}}{1+M}
$$

with

$$
\beta_{0}=\frac{1}{2 \sqrt{2}} \frac{L_{p}}{S_{p}} \sqrt{\frac{\omega \nu}{c^{2}}}\left(1+\frac{\gamma-1}{\sqrt{P r}}\right)
$$

where $\nu$ is the kinematic viscosity of air, $L_{p}$ is the perimeter of the duct cross-section, $S_{p}$ is the duct cross-sectional area and $\mathrm{Pr}$ is the Prandtl number. The unknown pressure amplitudes, $p^{ \pm}$, are evaluated by measuring the pressure data at at least two positions. In the experiments, the measured data is obtained from four microphones each, placed on either side of the sample. Using Eq. (18), the upstream and downstream pressure amplitudes can be evaluated as

$$
\left[\begin{array}{ll}
\exp \left(i k^{+} x_{1,2}^{1}\right) & \exp \left(-i k^{-} x_{1,2}^{1}\right) \\
\exp \left(i k^{+} x_{1,2}^{2}\right) & \exp \left(-i k^{-} x_{1,2}^{2}\right) \\
\exp \left(i k^{+} x_{1,2}^{3}\right) & \exp \left(-i k^{-} x_{1,2}^{3}\right) \\
\exp \left(i k^{+} x_{1,2}^{4}\right) & \exp \left(-i k^{-} x_{1,2}^{4}\right)
\end{array}\right]\left[\begin{array}{l}
p_{1,2}^{+} \\
p_{1,2}^{-}
\end{array}\right]=\left[\begin{array}{l}
p\left(x_{1,2}^{1}\right) \\
p\left(x_{1,2}^{2}\right) \\
p\left(x_{1,2}^{3}\right) \\
p\left(x_{1,2}^{4}\right)
\end{array}\right]
$$

The superscript of $x$ denotes the microphone number, and the subscripts 1 and 2 indicate the upstream side and downstream side, respectively. The overdetermined system in Eq. (21) is solved with the help of Moore-Penrose pseudo inverse (see [30]) to obtain the unknown pressure amplitudes $p_{1}^{+}, p_{2}^{+}, p_{1}^{-}$and $p_{2}^{-}$.

This method relies on the acoustic field being one-dimensional. It is safe to assume that this is the case, provided that the acoustic wavelength is at least twice as long as the larger dimension of the cross-section of the aluminium duct. In our case this is $120 \mathrm{~mm}$, allowing us to go up to a frequency of about $1400 \mathrm{~Hz}$. We measured the scattering matrix of both samples using a stepped sine excitation with frequencies between $100 \mathrm{~Hz}$ and $1400 \mathrm{~Hz}$.

\section{Experimental validation of the models}

\subsection{Huang and Heckl model for the tube row without cross-flow}

In order to put the Huang and Heckl model (Section 2.1) to the test, we measured the transmission and reflection coefficients for both samples without flow, as a function of 
frequency. The results for the magnitude of these coefficients are shown in Fig. 8, and those for the phase are shown in Fig. 9. In both figures, the markers give the experimental results, and the theoretical results have been added as continuous curves. The agreement is clearly good, not only for sample $d_{16}$ (row of 5 tubes), but also for sample $d_{20}$ (two half-cylinders). We can therefore conclude, that the Huang and Heckl model, which has been derived for a tube row, is also valid for similar geometries, like sample $d_{20}$.

The results in Fig. 8 show that with increasing frequency, the transmission coefficients decrease in magnitude, whereas the reflection coefficients increase. This is as one would expect, given that long waves (low frequency) are less hindered than short waves (high frequency) when propagating through constrictions (see chapter 3 in [31]). When the frequency reaches values, where the wavelength becomes as small as the tube spacing $h_{p}$, diffraction occurs. For the geometry of our sample $d_{16}$, this would happen at $17,000 \mathrm{~Hz}$, which is well beyond the frequency range we are considering.
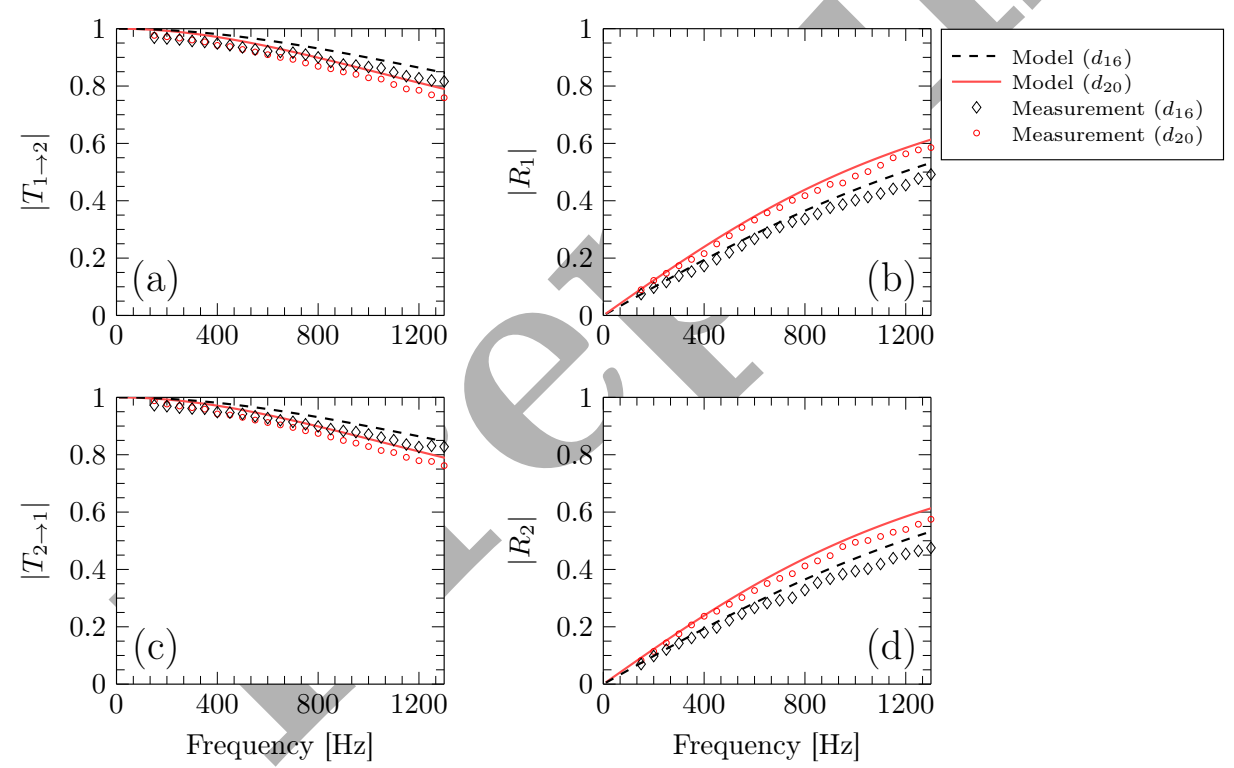

Figure 8: Magnitude of transmission and reflection coefficients for the two samples with no cross-flow. 

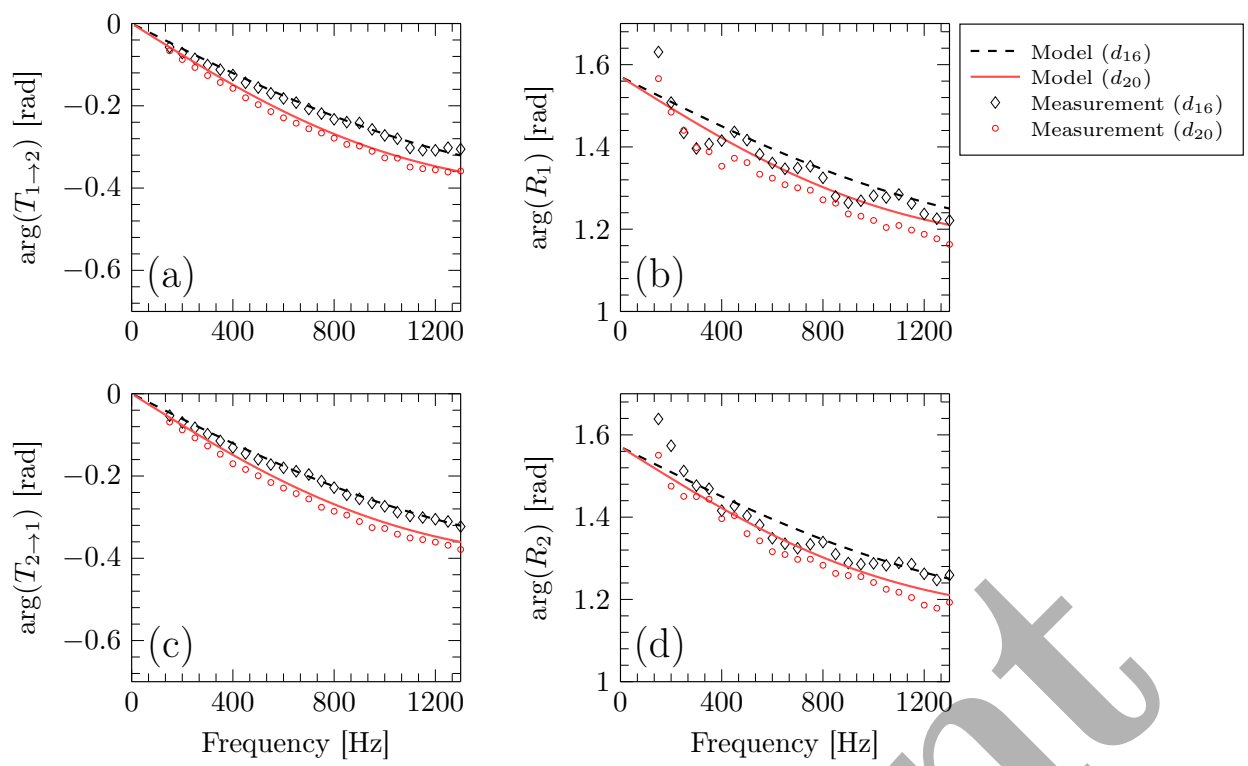

Figure 9: Phase of transmission and reflection coefficients for the two samples with no cross-flow.

The phase of the transmission coefficients is close to zero for low frequencies; this is in line with our earlier observation that the samples are "transparent" to low-frequency acoustic waves.

\subsection{Quasi-steady model for the tube row with cross-flow}

In order to validate our quasi-steady model, we tested the two samples for seven incoming velocities: $u_{1}=5.0,7.5,9.5,11.5,13.5,14.5$ and $15.5 \mathrm{~m} / \mathrm{s}$. Figures 10 and 11 show the predicted (continuous curves) as well as measured (markers) $\left|T_{1 \rightleftarrows 2}\right|$ and $\left|R_{1,2}\right|$ (coefficients for total enthalpy) versus Mach number $\left(M_{1}\right)$; three frequencies were considered: $f=150$, 250 and $350 \mathrm{~Hz}$, for $d_{16}$ and $d_{20}$ respectively. The theoretical predictions correspond to those shown in Fig. 6. We observe that the predicted results agree well with the measurements, for both samples. Moreover, the measured values barely change with frequency; this confirms that the behaviour is indeed quasi-steady for the frequencies and velocities we considered.

Figure 10 also proves that the quasi-steady model, which has been derived for the halfcylinders, is valid for tube rows $\left(d_{16}\right)$. The frequencies shown in Figs. 10 and 11 are chosen based on the Strouhal and Helmholtz number criterion discussed in Section 6.3 . 

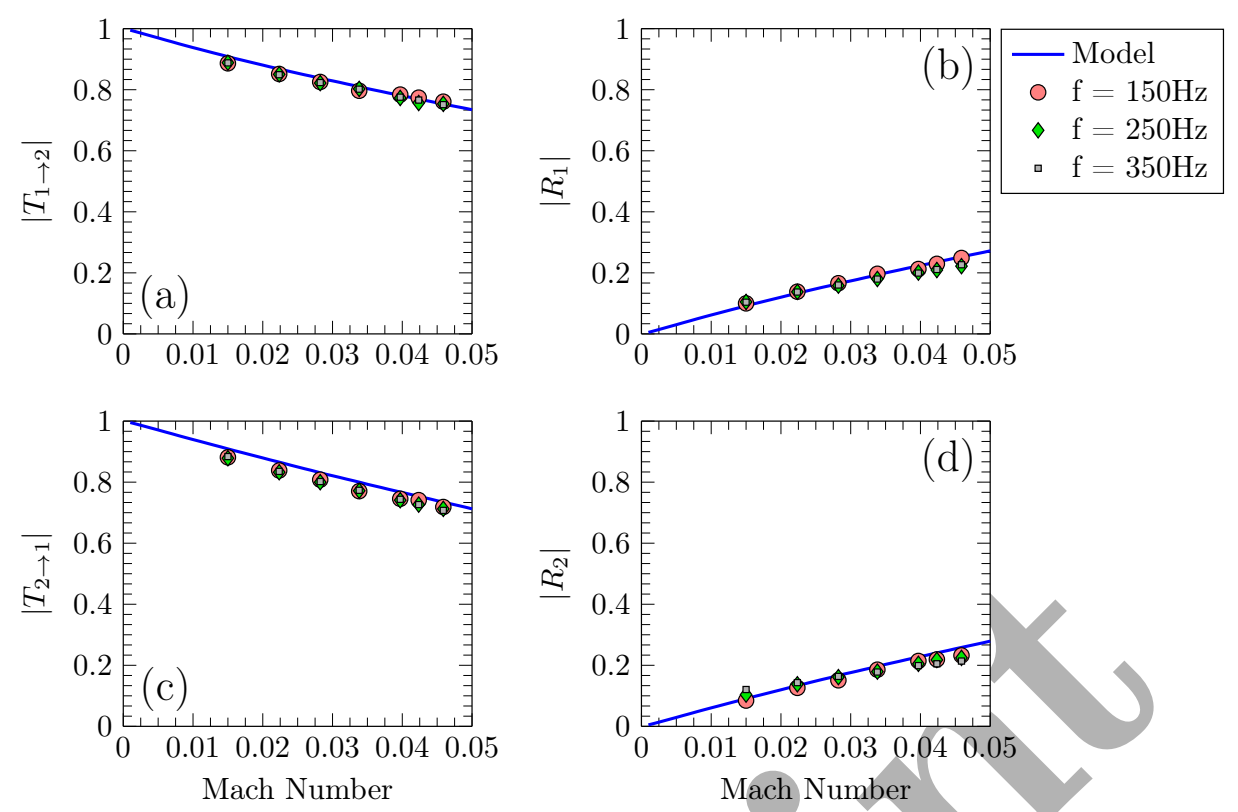

Figure 10: Magnitude of transmission and reflection coefficients (of total enthalpy) for $d_{16}$ with cross-flow.
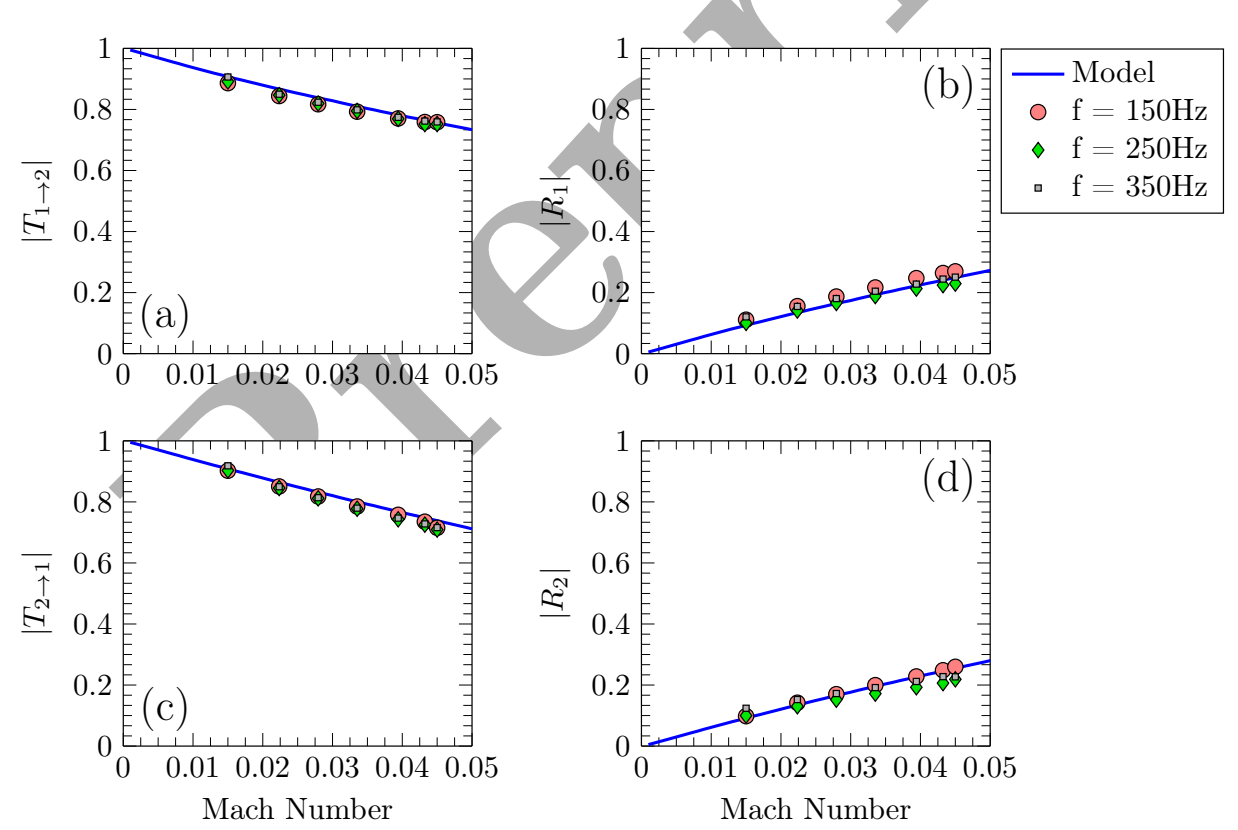

Figure 11: Magnitude of transmission and reflection coefficients (of total enthalpy) for $d_{20}$ with cross-flow.

\subsection{Limit of validity of the quasi-steady model}

In this section, we use our experimental results to explore the limits of validity of our quasi-steady model in terms of frequency and Strouhal number. Figures 12 and 13 show the measured reflection and transmission coefficients (of total enthalpy) as a function of frequency, for the sample $d_{16}$ and $d_{20}$, respectively. The frequency range depicted is $0 \cdots 1200$ 
$\mathrm{Hz}$; data for higher frequencies have been discarded because transverse modes appear above $1420 \mathrm{~Hz}$ and the plane-wave assumption, which is essential for the multi-microphone measurements, breaks down. This means that we have a critical Helmholtz number, $H e=f h_{d} / c$, beyond which the measurements are not reliable. For $1420 \mathrm{~Hz}$ and $h_{d}=120 \mathrm{~mm}$, we can estimate the critical $\mathrm{He}$ as 0.5 . The mean flow velocities were: $u_{1}=5.0,7.5,11.5$ and $15.5 \mathrm{~m} / \mathrm{s}$. The markers in Figs. 12 and 13 denote the measured values. Also shown in these figures are the theoretical results (denoted by horizontal lines) from the quasi-steady model.

We observe that the measured values agree well with the theoretical values at low frequencies. The agreement depends on the mean velocity: for example, for $5.0 \mathrm{~m} / \mathrm{s}$ it is reasonable until about $600 \mathrm{~Hz}$ for $d_{16}$ and $450 \mathrm{~Hz}$ for $d_{20}$, whereas for $11.5 \mathrm{~m} / \mathrm{s}$ it is reasonable until about $1200 \mathrm{~Hz}$ for $d_{16}$ and $1000 \mathrm{~Hz}$ for $d_{20}$. This suggests that the Strouhal number, $S t=f /\left(u_{g} / r\right)$, plays an important role in quantifying the range of agreement. Figures 14 and 15. which show, respectively, the results of Figs. 12 and 13 in terms of Strouhal number, rather than frequency, confirm this suggestion. The agreement is now rather more uniform for the different bias-flow velocities.

In order to estimate a Strouhal number range for the quasi-steady model, we choose (by visual inspection of $T$ coefficients) frequency limits from the measurements, for the various velocities considered. For small mean flow velocities, we choose those frequency values as limiting values when the measurements start to deviate significantly from the quasi-steady model predictions. These are termed quasi-steady limits, and are denoted by solid markers in Fig. 16. Figure 16 depicts the frequency limits as plotted against $\left(u_{g} / r\right)$. We can observe that the frequency limits increase with increasing velocity. Beyond a certain velocity (say $13.5 \mathrm{~m} / \mathrm{s}$ ), the frequency limits (hollow markers in Fig. 16) are constant values, at $1200 \mathrm{~Hz}$ (corresponding to $\mathrm{He}=0.42$ ). This is because the measurements beyond $1200 \mathrm{~Hz}$ are polluted by the transverse mode at $1420 \mathrm{~Hz}$ and could no longer be considered acceptable for estimating the valid range for the quasi-steady model. These limits are termed transverse mode limits. This suggests that we could have two limits for the validity of our model: one pertaining to the quasi-steady assumption (low Strouhal number) and the other pertaining to the breakdown of plane wave assumption (low Helmholtz number).

The Strouhal number limit for our study is depicted as solid the line in Fig. 16, whereas the Helmholtz number limit is depicted as the broken line. For low velocities, where the plane wave assumption holds, we can estimate the $S t$ limit as 0.16. As expected, this value satisfies the assumption $S t \ll 1$ made in Section 2.2.1. However, for high velocities where the plane wave assumption breaks down, we can estimate the $\mathrm{He}$ limit to be 0.42 (see Fig. 16), 
which satisfies the assumption of $(H e)^{2} \ll 1$. This limit is a consequence of the duct shape (cross-section) and can be varied by varying the duct shape.
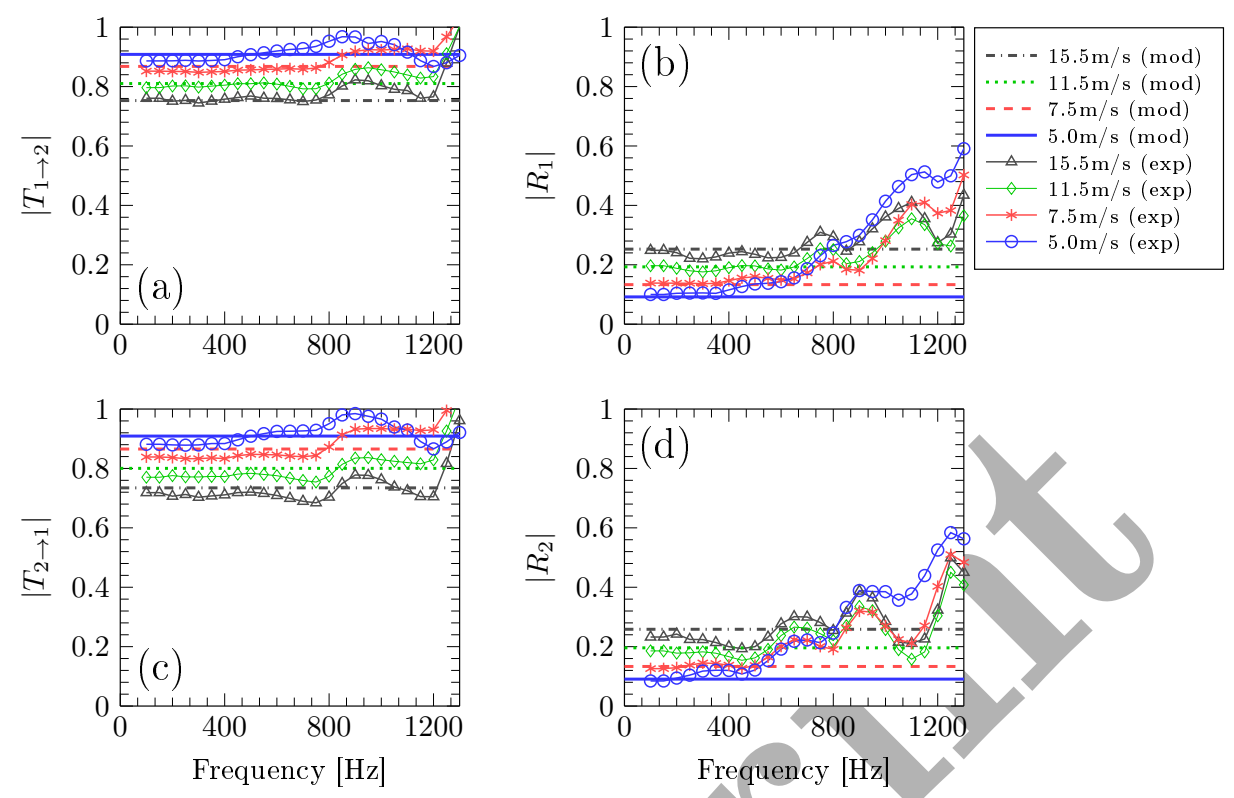

Figure 12: Magnitude of transmission and reflection coefficients (of total enthalpy) versus frequency for $d_{16}$ and different cross-flow velocities.
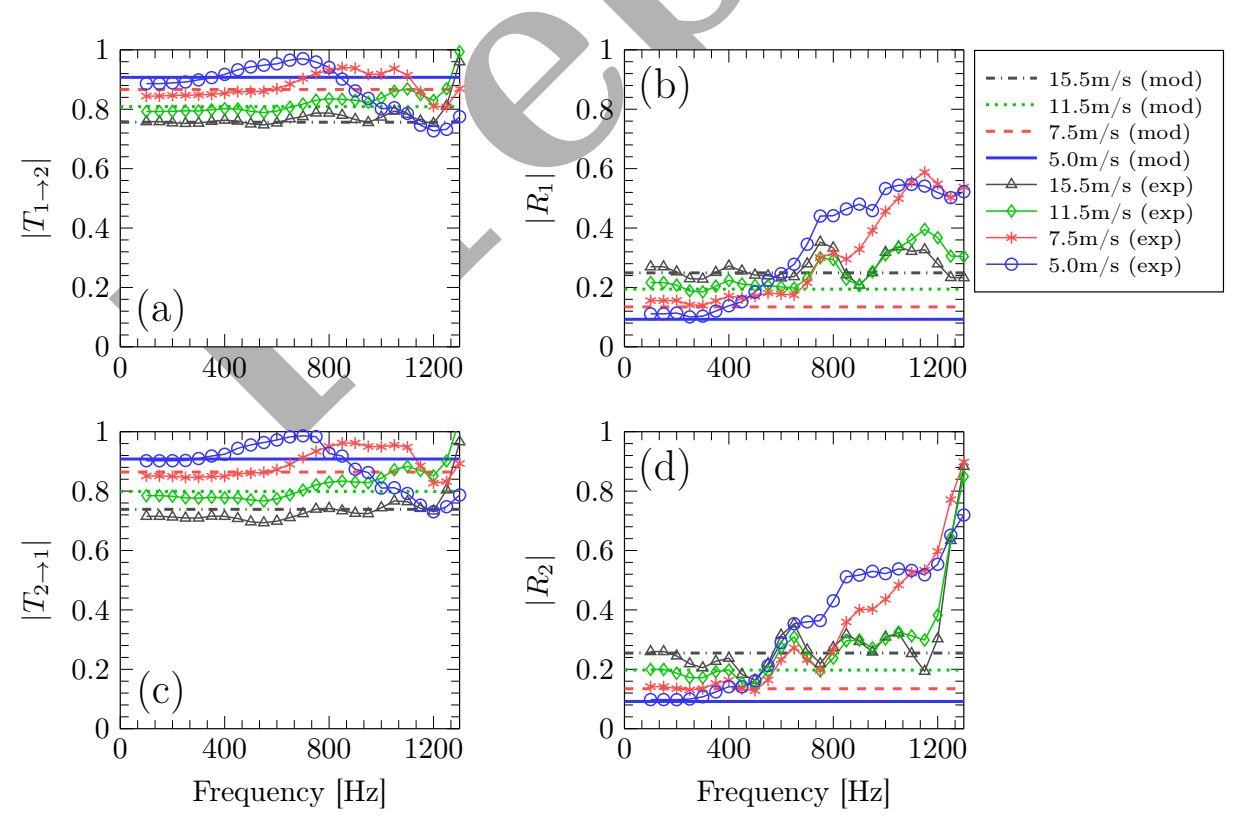

Figure 13: Magnitude of transmission and reflection coefficients (of total enthalpy) versus frequency for $d_{20}$ and different cross-flow velocities. 

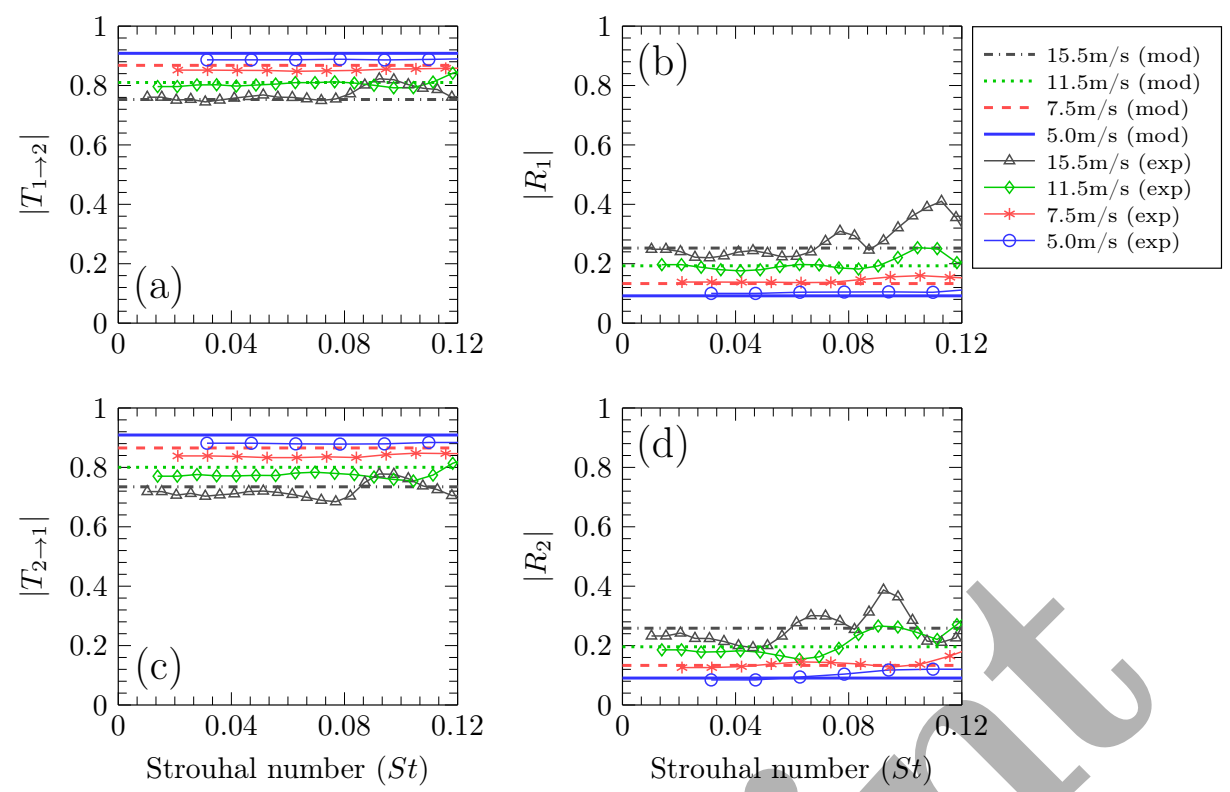

Figure 14: Magnitude of transmission and reflection coefficients (of total enthalpy) versus Strouhal number for $d_{16}$ and different cross-flow velocities.

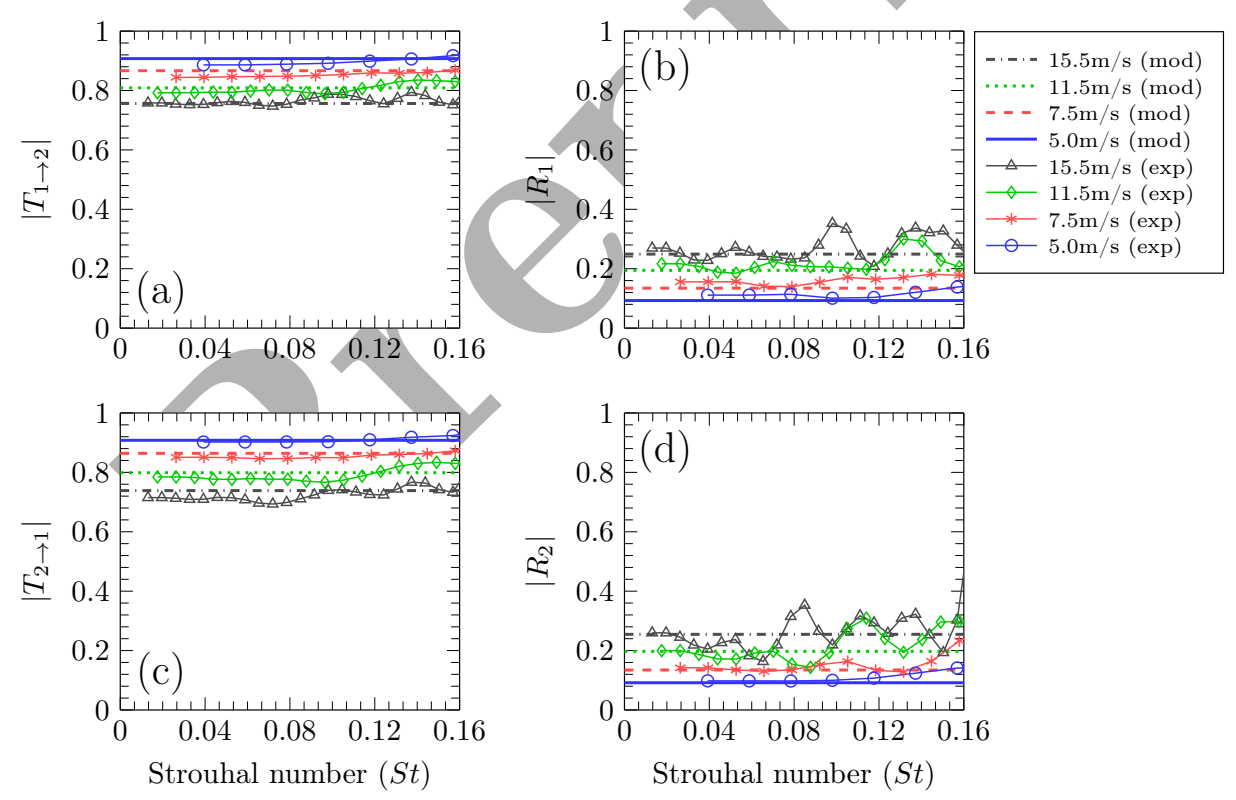

Figure 15: Magnitude of transmission and reflection coefficients (of total enthalpy) versus Strouhal number for $d_{20}$ and different cross-flow velocities.

\section{Conclusion}

In the present study, we examined the aeroacoustic response of an array of circular tubes, both theoretically and experimentally. For the case without bias-flow, the theoretical model of Huang and Heckl [9] was used. Its predictions for the reflection and transmission co- 


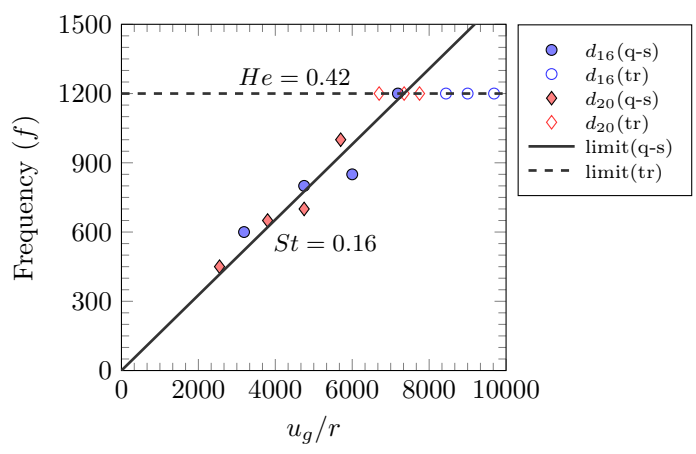

Figure 16: Frequency limits vs. $u_{g} / r$, for the various velocities considered. The solid markers denote the quasi-steady limit and the hollow markers denote the transverse mode limit. The solid line is the $S t$ limit for the quasi-steady assumption and the broken line is the $H e$ limit for the plane wave assumption.

efficients agree well with the corresponding measured results, which we obtained using a multi-microphone technique. We have hence validated this model.

For the case with bias-flow, we developed a new theoretical model, based on a quasisteady flow assumption, and then used this model to predict the reflection and transmission coefficients for several bias-flow velocities. In order to validate this model, we measured the corresponding coefficients and identified the critical values of frequency and Strouhal number, where the agreement started to break down, both in terms of the quasi-steady assumption and the plane wave assumption. From this we deduced the limit of validity of our quasi-steady model in terms of Strouhal number, $S t=f r / u_{g}<0.16$, using the quasi-steady assumption, and Helmholtz number, $H e=f h_{d} / c<0.42$, using plane-wave assumption. Both these values satisfy our initial criteria of $S t \ll 1$ and $(H e)^{2} \ll 1$.

The results from the quasi-steady model approach those of the Huang and Heckl model in the limit where the bias-flow velocity tends to zero; thus the two models are consistent.

We considered two different samples with the same open area ratio: (i) cylinders with small diameter $(d=16 \mathrm{~mm})$ and small gaps between them $\left(h_{g}=4 \mathrm{~mm}\right)$, and (ii) cylinders with larger diameter $(d=20 \mathrm{~mm})$ and larger gaps between them $\left(h_{g}=5 \mathrm{~mm}\right)$. The reflection and transmission coefficients were the same for both samples, indicating that the geometry can be scaled in terms of the open area ratio.

Our theoretical model is only valid for low frequencies (plane waves) and low Strouhal numbers (quasi-steady). Nevertheless, it is a valuable tool for designers of heat exchangers in combustion systems. In a domestic boiler, for example, the frequency of a thermoacoustic instability is typically a few hundred Hertz. It is therefore quite feasible that a carefully 
designed heat exchanger acts as an acoustic sink, which counteracts the sound generation by the flame.

\section{Acknowledgements}

The presented work is part of the Marie Curie Initial Training Network Thermo-acoustic and Aero-acoustic Nonlinearities in Green combustors with Orifice structures (TANGO). We gratefully acknowledge the financial support from the European Commission under call FP7-PEOPLE-ITN-2012. We are also grateful to Dr. Xavier Pelorson, (CNRS, GIPSA-Lab, Grenoble) for helping us implement the Thwaites' method.

\section{Appendix A. Calculation of the flow separation point}

\section{Appendix A.1. Thwaites' Method}

In order to find the flow separation point on the cylinder, we start with the integral form of the boundary layer equations, known as the von Kármán momentum integral equation (see Eq. A.1 ), which involves the wall shear stress $\left(\tau_{0}\right)$, the displacement $\left(\delta^{*}\right)$ and momentum $(\Theta)$ thicknesses of the boundary layer.

$$
U_{e} \delta^{*} \frac{\mathrm{d} U_{e}}{\mathrm{~d} x}+\frac{\partial}{\partial x}\left(U_{e}^{2} \Theta\right)=\frac{\tau_{0}}{\rho}
$$

where $U_{e}(x)$ denotes the bulk flow velocity at the edge of the boundary layer.

Thwaites (1949) 32 developed an approximate solution to the von Kármán equation by using shape parameters that depend only on the boundary layer properties $\tau_{0}, \delta^{*}$ and $\Theta$. These shape parameters are defined as,

$$
\mathcal{H}=\frac{\delta^{*}}{\Theta}
$$

and

$$
\mathcal{T}=\frac{\Theta}{\nu U_{e}} \frac{\tau_{0}}{\rho}
$$

The von Kármán equation (Eq. A.1p) can now be written in terms of the only unknown variable $\Theta$ as,

$$
\frac{\mathrm{d}}{\mathrm{d} x}\left(\frac{\Theta^{2}}{\nu}\right)+\frac{2}{U_{e}}\left[(\mathcal{H}+2) \frac{\mathrm{d} U_{e}}{\mathrm{~d} x}\left(\frac{\Theta^{2}}{\nu}\right)-\mathcal{T}\right]=0 .
$$

Thwaites gave the following approximation to the shape parameters

$$
2(\mathcal{H}+2) \lambda-2 \mathcal{T}=6 \lambda-0.45,
$$


where $\lambda=\frac{\mathrm{d} U_{e}}{\mathrm{~d} x}\left(\frac{\Theta^{2}}{\nu}\right)$. Substituting Eq. A.5. in Eq. A.4. and integrating it along the axial direction $(x)$ gives

$$
\Theta^{2}=\frac{0.45 \nu}{U_{e}^{6}(x)} \int_{0}^{x} U_{e}^{5}(\tilde{x}) \mathrm{d} \tilde{x}+\frac{\Theta_{0}^{2} U_{0}^{6}}{U_{e}^{6}(x)}
$$

Here, $\tilde{x}$ is the integration variable and $\Theta_{0}$ and $U_{0}$ are the values of $\Theta$ and $U_{e}$ at $x=0$, respectively. If $x=0$ is a stagnation point, then $U_{e}=U_{0}=0$ and $\Theta=\Theta_{0}=0$. Once $\Theta$ is evaluated, we can calculate $\lambda$ and the shape parameters $\mathcal{H}$ and $\mathcal{T}$ from Fig. A.1, the data for which is provided in 32 . We can now use these parameters to find the flow separation location on the cylinder surface, as shown in the next section.

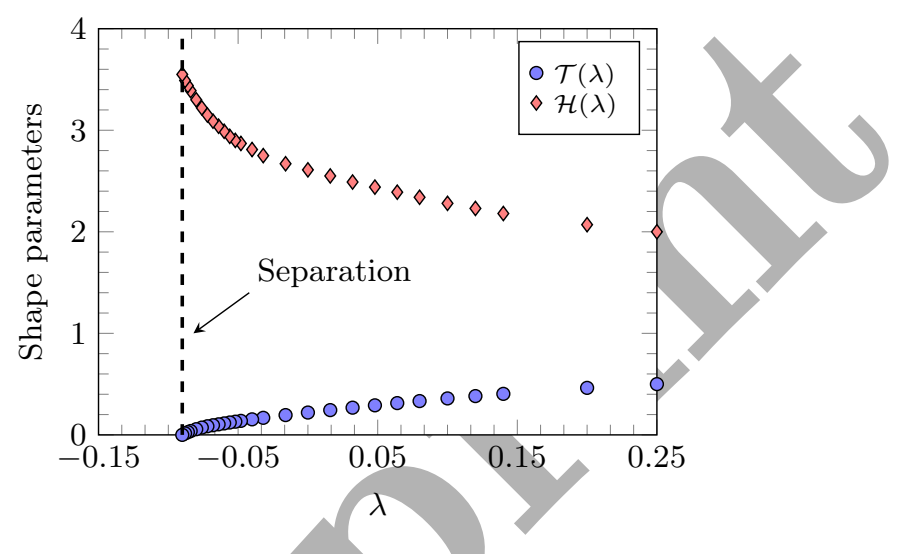

Figure A.1: Plot for the shape factors $\mathcal{H}$ and $\mathcal{T}$ vs. $\lambda$ used in Thwaites' method.

\section{Appendix A.2. Flow separation location}

Flow separation is the onset of flow reversal on the solid surface and the flow will no longer be attached to the surface. At the separation point on the wall, the surface shear stress will go to zero. This implies that $(\partial u / \partial y)_{\text {wall }}=0$, or in terms of the shape parameter, $\mathcal{T}=0$. This will give the criterion for flow separation as $\lambda_{S}=-0.0992^{1}$ (subscript ' $S$ ' indicates separation).

In order to find the separation location on the hex cylinder surface, we discretize the flow domain into $N$ divisions of interval $\Delta x$. The grid points are numbered sequentially and denoted by the subscript $i$. The algorithm for finding the separation point is as follows [14]:

1. Initialise $U_{e, 1}=U_{0}, \Theta_{1}=0, \mathcal{H}_{1}=0$ and $\lambda_{1}=0$.

\footnotetext{
${ }^{1}$ The separation criterion of $\lambda_{S}=-0.0992$ is an adaptation from 33. The original criterion used by Thwaites is $\lambda_{S}=-0.09$.
} 
2. Evaluate

$$
\begin{aligned}
U_{e, i} & =\frac{U_{e, 1} h_{1}}{h_{i}-2 \mathcal{H}_{i-1} \Theta_{i-1}} \\
\Theta_{i} & =\sqrt{\frac{0.45 \nu \Delta x \sum_{j=1}^{i} U_{e, j}^{5}}{U_{e, i}^{6}}} \\
\lambda_{i} & =\frac{\Theta_{i}^{2}}{\nu} \frac{U_{e, i}-U_{e, i-1}}{\Delta x}
\end{aligned}
$$

where $h_{i}$ is the height of the duct at any location $x_{i}$ along the flow direction.

3. Once $\lambda_{i}$ is known, evaluate $\mathcal{H}\left(\lambda_{i}\right)$ from Fig. A.1

4. Using a relaxation scheme, iterate for $U_{e, 1}, \Theta_{i}, \lambda_{i}$ and $\mathcal{H}_{i}$, till they converge.

5. Check for flow separation by comparing the value of $\lambda_{i}$ with $\lambda_{S}$.

(a) If $\lambda_{i} \neq \lambda_{S}$, move to the next grid point and repeat steps 2 to 5 .

(b) If $\lambda_{i}=\lambda_{S}$, compute the location of separation for the corresponding $x_{i}$.

Appendix A.3. Variation of jet height $\left(h_{j}\right)$ with Mach number $\left(M_{1}\right)$

The jet height $\left(h_{j}\right)$ can be easily computed once the separation point, $x_{s}$, is found using the algorithm given in Appendix A.2. Figure A.2 shows the variation of normalised jet height $\left(h_{j} / h_{g}\right)$ with varying incoming Mach number $\left(M_{1}\right)$, for the configuration of two halfcylinders of diameter $d=20 \mathrm{~mm}$ and separated by a gap height $h_{g}=5 \mathrm{~mm}$. The curve shows that for the Mach number range considered, $h_{j} / h_{g}$ is always greater than 1 indicating that the separation always occurs downstream of the throat region. Moreover, separation is controlled by yiscosity (Reynolds number $R e_{g}=u_{g} h_{g} / \nu$ ) rather than by compressibility (Mach number) and the Thwaites' method is valid only for laminar boundary layers. Hence, one must be careful when applying this method to large Mach numbers $\left(M_{1}=R e_{g} \nu /[(d+\right.$ $\left.\left.h_{g}\right) c\right]$ ) where the model might fail due to transition to turbulent boundary layers.

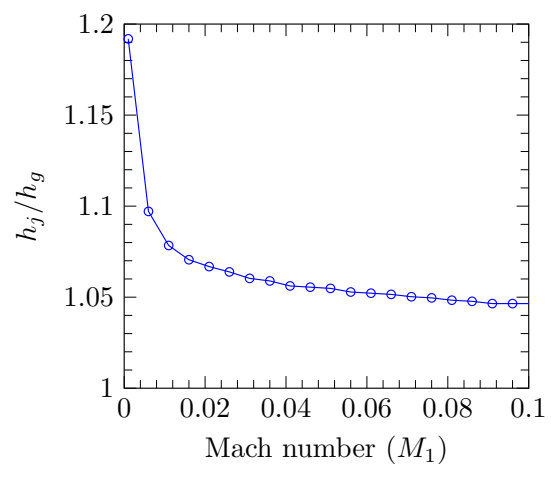

Figure A.2: Variation of normalised jet height with Mach number. 


\section{References}

[1] P. Mungur, F. J. Fahy, Transmission of sound through an array of scatterers, J. Sound Vib. 9 (2) (1969) 287-294.

[2] U. R. Kristiansen, F. J. Fahy, Scattering of Acoustic waves by an N-layer periodic grating, J. Sound Vib. 24 (3) (1972) 315-335.

[3] C. M. Linton, D. V. Evans, The interaction of waves with a row of circular cylinders, J. Fluid Mech. 251 (1993) 687-708.

[4] M. A. Heckl, Sound Propagation in Bundles of Periodically Arranged Cylindrical Tubes, Acustica 77 (3) (1992) $143-152$.

[5] M. A. Heckl, L. S. Mulholland, Some recent developements in the theory of acoustic transmission in tube bundles, J. Sound Vib. 179 (1) (1995) 37-62.

[6] V. Twersky, On Scattering of Waves by the Infinite Grating of Circular Cylinders, IRE Trans. Anntenas Propag. 10 (1962) 737-765.

[7] V. Twersky, On the Scattering of Waves by an Infinite Grating, IRE Trans. Anntenas Propag. 4 (1956) 330-345.

[8] V. Twersky, Elementary function representations of Schlömilch series, Arch. Ration. Mech. Anal. 8 (1) (1961) $323-332$.

[9] X. Y. Huang, M. A. Heckl, Transmission and Dissipation of Sound Waves in Tube Bundles, Acustica 78 (4) (1993) $191-200$.

[10] M. C. Quinn, M. S. Howe, The Influence of Mean Flow on the Acoustic Properties of a Tube Bank, P. Roy. Soc. A-Math. Phy. 396 (December) (1984) 383-403.

[11] A. P. Dowling, I. J. Hughes, Sound absorption by a screen with a regular array of slits, J. Sound Vib. 156 (3) (1992) 387-405.

[12] G. C. J. Hofmans, G. Groot, M. Ranucci, G. Graziani, A. Hirschberg, Unsteady flow through in-vitro models of the glottis, J. Acoust. Soc. Am. 113 (3) (2003) 1658-1675.

[13] M. Deverge, X. Pelorson, C. Vilain, P. Y. Lagrée, F. Chentouf, J. Willems, A. Hirschberg, Influence of collision on the flow through in-vitro rigid models of the vocal folds, J. Acoust. Soc. Am. 114 (6) (2003) 3354-3362, part 1.

[14] C. E. Vilain, X. Pelorson, C. Fraysse, M. Deverge, A. Hirschberg, J. Willems, Experimental validation of a quasi-steady theory for the flow through the glottis, J. Sound Vib. 276 (2004) 475-490. 
[15] D. Ronneberger, Experimentelle Untersuchungen zum akustischen Reflexionsfaktor von unstetigen Querschnittsänderungen in einem luftdurchströmten rohr (Experimental Investigations about the Acoustic Reflection Coefficient of Discontinuous Changes of Cross-Section in Tubes with Air Flow), Acustica 19 (1967/68) $222-235$.

[16] Y. A. Çengel, J. M. Cimbala, Fluid Mechanics: Fundamentals and Applications, 1st Edition, McGraw-Hill series in mechanical engineering, 2006.

[17] R. C. Mottram, The behaviour of orifice and venturi-nozzle meters in pulsating flow, Ph.D. thesis, University of Surrey (1971).

[18] F. Schultz-Grunow, Durchflußmeßverfahren für pulsierende Strömungen (Flow measurement methods for pulsating flows), Forschung auf dem Gebiete des Ingenieurwesens 12 (3) (1941) $117-126$.

[19] G. C. J. Hofmans, R. J. J. Boot, P. P. J. M. Durrieu, Y. Auregan, A. Hirschberg, Aeroacoustics response of a slit-shaped diaphragm in a pipe at low Helmholtz number, 1: Quasi-steady results, J. Sound Vib. 244 (1) (2001) 35-56.

[20] G. C. J. Hofmans, Vortex Sound in Confined Flows, Ph.D. thesis, Technische Universiteit Eindhoven (1998).

[21] S. Rienstra, A. Hirschberg, An Introduction to Acoustics, Tech. Rep. IWDE 92-06, Eindhoven University of Technology (2017).

[22] E. Motheau, L. Selle, F. Nicoud, Accounting for convective effects in zero-Mach-number thermoacoustic models, J. Sound Vib. 333 (1) (2014) 246-262.

[23] P. E. Doak, Fluctuating Total Enthalpy as the Basic Generalized Acoustic Field, Theor. Comput. Fluid Dyn. 10 (1998) 115-133.

[24] A. Surendran, Passive control of thermoacoustic instabilities in idealised combustion systems using heat exchangers, Ph.D. thesis, Keele University (2017).

[25] L. Zhou, Acoustic characterization of orifices and perforated liners with flow and highlevel acoustic excitation, Ph.D. thesis, KTH Royal Institute of Technology, Stockholm (2015).

[26] L. Peerlings, Methods and techniques for precise and accurate in-duct aero-acoustic measurements : Application to the area expansion, Licentiate thesis, Royal Institute of Technology, Stockholm (2015). 
[27] M. Åbom, Measurement of the scattering-matrix of acoustical ports, Mech. Syst. Sig. Process. 5 (2) (1991) 89-104.

[28] S.-H. Jang, J.-G. Ih, On the multiple microphone method for measuring in-duct acoustic properties in the presence of mean flow, J. Acoust. Soc. Am. 103 (3) (1998) 1520-1526.

[29] A. D. Pierce, Acoustics: An Introduction to Its Physical Principles and Applications, Acoustical Society of America, 1994.

[30] G. W. Stewart, J.-g. Sun, Matrix Perturbation Theory, 1st Edition, Academic Press Inc., 1990.

[31] A. P. Dowling, J. E. Ffowcs Williams, Sound and Sources of Sound, Ellis Horwood Limited, 1983.

[32] P. K. Kundu, I. M. Cohen, D. R. Dowling, Fluid Mechanies, 5th Edition, Elsevier Inc., 2012.

[33] X. Pelorson, A. Hirschberg, R. R. van Hassel, A. P. J. Wijnands, Y. Auregan, Theoretical and experimental study of quasisteady-flow separation within the glottis during phonation. Application to a modified two-mass model, J. Acoust. Soc. Am. 96 (6) (1994) $3416-3431$. 